\title{
I NFLUENCIA DE LAS CREENCIAS ANDROCÉNTRICAS EN LA VIVENCIA DE LA MATERNIDAD EN MUJERES DE UNA COMARCA RURAL (ESPAÑA)
}

\section{THE INFLUENCE OF ANDROCENTRIC BELIEFS ABOUT THE MATERNITY EXPERIENCE IN A RURAL REGION (SPAIN)}

Autores:

Torres Luzón, Cristina ${ }^{a}$

Romo Avilés, Nuria ${ }^{b}$

Gil García, Eugenia ${ }^{c}$

a Unidad Materno Infantil. Hospital de Guadix.

b Instituto de Estudios de la Mujer. Universidad de Granada.

c Facultad de Enfermería, Fisioterapia y Podología. Universidad de Sevilla.

Correspondencia: ctluzon@gmail.com 


\section{RESUMEN}

Título

Influencia de las creencias androcéntricas en la vivencia de la maternidad en mujeres de una comarca rural (España)

\section{I ntroducción}

La maternidad esta impregnada de creencias androcéntricas que ponen en riesgo a las mujeres. Estos riesgos se basan en las sobrecargas físicas, psicológicas y emocionales que se producen en las madres.

\section{Objetivo}

Conocer cómo influyen las creencias androcéntricas de un grupo de mujeres rurales en su proceso de maternidad.

\section{Método}

Investigación cualitativa a través de 11 entrevistas en profundidad realizadas a las puérperas del hospital de Guadix. Los datos fueron analizados con el método descubrimiento, codificación y relativización.

\section{Resultados}

Las mujeres presentan sentimientos de miedo, culpa e inseguridad. La figura paterna no se involucra en la crianza ni en el hogar. La red de apoyo a las madres la forman sólo mujeres, generalmente de la familia.

\section{Discusión-Conclusión}

La perspectiva de género muestra las limitaciones y sobrecargas que producen las creencias androcéntricas en las madres. Dichas creencias merman la calidad de vida y ponen en riesgo la salud de las mujeres. Aportación para enfermería

El personal de enfermería debe conocer cómo afecta la mentalidad androcéntrica al proceso de maternidad. Debe detectar los factores que vulneran a las mujeres en su vivencia, además de capacitar a ambos sexos para un reparto igualitario de roles.

Palabras clave: conducta materna, factores de riesgo, identidad de género, período de postparto, Enfermería. 


\section{ABSTRACT}

Title: The influence of androcentric beliefs about the maternity experience in a rural region.

\section{I ntroduction}

The maternity is influenced by the androcentric beliefs which put women in risk. These risks are based on physical, psychological and emotional overload that are produced in women.

\section{Objective}

To know how a rural woman group are influenced by androcentric beliefs during their maternity process.

\section{Method}

Qualitative research through interviews with 11 women who has just given birth Guadix Hospital. The data were analyzed using the method of discovering, coding and relativity.

\section{Result}

The women present feelings of fear, guilt and insecurity. The father figure in not involve in the upbringing of the child nor in the home. Mothers only receive help from the women of their family.

\section{Discussion/ Conclusion}

The gender perspective shows the limitations and overloads which produce androcentric beliefs in mothers. Said beliefs reduce the quality of life and put the health of mothers at risk.

\section{Application for qualified nurse}

The hospital ward staff should know how the androcentric mentality affects the process of maternity. They should detect the factors which made women vulnerable in their experience, in addition they should teach both sexes to an equal distribution of roles.

Key words: maternal behaviour, risk factors, gender identity, postpartum period, Nursing. 


\section{NTRODUCCIÓN}

En las últimas décadas la maternidad está siendo estudiada a través de la perspectiva de género. Uno de los objetivos es mostrar las desigualdades existentes y poder dar una mayor libertad de decisión a las mujeres.

El concepto maternidad se ha ido construyendo en cada época, en función de las necesidades e ideologías de cada sociedad. La maternidad fue establecida como eje vertebral de la identidad femenina. Sin embargo, muchas mujeres hoy en día se están cuestionando esta "parte inherente" de su identidad.

Acceder a la maternidad por la inercia social irreflexiva, sin un proceso de autodeterminación consciente, puede acabar ocasionando perjuicios tanto para las madres como para su entorno ${ }^{1}$.

Analizando las connotaciones históricas del concepto maternidad vemos como las sociedades han establecido e inculcado en las mujeres patrones de comportamiento maternal. Estos comportamientos son asociados a ellas de una manera "natural" e "instintiva" a través de mitos, creencias y estereotipos como son el amor maternal, instinto maternal, maternidad intensiva, "la buena madre"(2,1,3,4,5). Toda esta ideología androcéntrica ha tenido como objetivo relegar y perpetuar a las mujeres al ámbito de lo privado, para realizar los trabajos sociales no remunerados como son el mantenimiento del hogar, la crianza y el cuidado de los adultos. Estas creencias se han ido transmitiendo a lo largo de los siglos, sin embargo, con la incorporación de las mujeres al mundo laboral y social, estas creencias latentes están ocasionando en la mayoría de las mujeres diferentes malestares psico-emocionales, además de sobrecargas a nivel físico debido al reparto desigual de roles.

El proceso de la maternidad, como es vivido actualmente, conlleva una serie de riesgos ocultos para la salud de las mujeres como son la labilidad

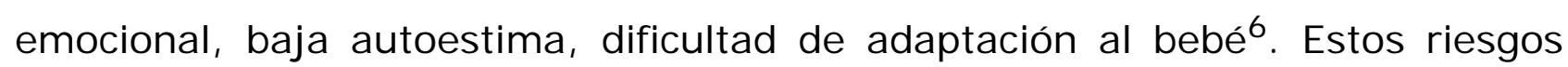
hacen peligrar su bienestar y calidad de vida, además de mermar y distorsionar sus procesos de desarrollo personal. La aplicación de la 
perspectiva de género permite mostrar la influencia de estas creencias que privan a las mujeres de vivir una maternidad propia y saludable.

\section{ANTECEDENTES}

Existen múltiples estudios, a nivel nacional e internacional, sobre los factores de riesgo de la depresión postparto7,8,9,10, los cuales varían en función de cada país y cultura. El riesgo de sufrir depresión postparto tiende a ser asociado a factores socioculturales y económicos como nivel educativo y clase socioeconómica baja.

Fuera de la temática depresión postparto, nos encontramos en la literatura científica estudios sobre la vivencia maternidad en circunstancias concretas como madres con cáncer o presas en cárceles $(11,12,13,14,15,16,17)$. Sin embargo, estas investigaciones no estudian la carga previa, que en si conlleva las creencias androcéntricas.

Pocos son los estudios encontrados que visibilicen la influencia que para las mujeres tiene la mentalidad androcéntrica sobre su identidad femenina y la maternidad $(6,18,19,20,21,22,23)$.

\section{OBJ ETIVO DE ESTUDIO}

Conocer cómo influyen las creencias androcéntricas sobre la vivencia de la maternidad en mujeres de ámbito rural.

\section{METODOLOGÍ A}

\section{Muestra}

El ámbito de estudio es la comarca rural de Guadix (Granada). Esta comarca presenta un alto grado de emigración por motivos laborales en los últimos años y una tasa de paro en mujeres del $44 \%$. Es una comarca con una actividad económica dividida en un $72 \%$ por el sector servicios, $15 \%$ de industria y $13 \%$ de construcción. El sector agrario ha descendido en la última 
década un 33\%. Estas características muestran la dificultad que presenta la comarca en estos tiempos, siendo un factor que repercute en las madres.

La muestra estuvo formada por 11 mujeres de dicha comarca.

Los criterios de inclusión fueron mujeres de nacionalidad española y ascendencia española, que parieron en el hospital de Guadix (Granada), encontrándose ingresadas por dicho motivo durante los meses de estudio (febrero y marzo del 2012) y que aceptaron participar en el estudio firmando el consentimiento informado.

Los criterios de exclusión fueron: madres solteras y/o adolescentes, madres tras cesárea, madres con recién nacidos patológicos y/o complicaciones en la madre tras el parto. También mujeres con dificultad idiomática y/o nacionalidad distinta a la española, y mujeres con auto identificación a una etnia, como por ejemplo la gitana. Los criterios de exclusión se basan en que el proceso de maternidad varía significativamente dependiendo de la presencia de alguno de estos condicionantes como refleja Ramona T. Mercer en su teoría “Comenzar a ser madre"24. Por otro lado, las creencias culturales varían dependiendo de la población, siendo este el motivo por el cual se restringió el tipo de mujeres que participaron en el estudio. En esos casos sería necesario otros estudios específicos donde se estudien esos factores y su efecto.

El tamaño de la muestra vino determinado por la profundidad y duración de la entrevista, la factibilidad para una única investigadora, así como la consecución de la saturación de la información en las categorías principales del estudio.

\section{Procedimiento}

Las entrevistas en profundidad fueron realizadas por la investigadora principal. Esta es enfermera con más de cuatro años de experiencia profesional en la unidad de hospitalización Materno Infantil. Durante el estudio la entrevistadora no trabajó en dicha unidad para evitar que las participantes pudieran sentirse condicionadas al ser atendidas por el mismo personal que las 
entrevistaba.

Las entrevistas se realizaron durante la estancia hospitalaria tras el parto, acordándose con ellas el momento para la entrevista.

La entrevistadora contó con la colaboración del personal de la Unidad Materno - Infantil, que avisó a la entrevistadora cuando valoraron que una madre era susceptible del estudio y deseaba participar.

Al inicio de la entrevista se les informó a través del consentimiento informado del objetivo del estudio, explicándoles que dicha información sería tratada de forma anónima y que se usaría una grabadora de voz para transcribir literalmente la conversación para su análisis.

Las entrevistas han sido grabadas, transcritas y analizadas por la investigadora principal.

Se usó un cuaderno de campo donde se anotó el lenguaje no verbal de las participantes.

\section{I nstrumento}

El guión de entrevista presentó un apartado de datos sociodemográficos y sanitarios, y otro con ítems sobre las variables de estudio (figura 1). El diseño se hizo para permitir realizar entrevistas abiertas, neutrales, sensibles y fáciles de comprender por la entrevistada.

\section{ANÁLI SIS DE LOS RESULTADOS}

La transcripción literal de la entrevista sirvió como una herramienta para asegurar la fiabilidad del análisis. Las entrevistas fueron analizadas de forma manual mediante el método descubrimiento, codificación y relativización empleado para este tipo de estudios.

Las categorías principales del estudio fueron deseo de la maternidad, implicación de la figura paterna, vivencia del embarazo, parto y puerperio, vida laboral, red de apoyo y cambios en la vida social de la puérpera tras la maternidad. 


\section{Posibles sesgos del estudio}

Un posible sesgo de recogida de información no diferencial se podía haber producido al estar presente en las entrevistas una grabadora de voz. Este sesgo se solucionó usando la grabadora de voz del móvil porque es vista como un elemento cotidiano. Además, antes de comenzar la entrevista, se dedicó un tiempo para familiarizarse con la mujer y que esta no se viera condicionada por la grabación.

Para evitar otro sesgo de recogida de información no diferencial las entrevistas siempre han sido realizadas por la misma persona basándose en el mismo guión de entrevista. Estas entrevistas, se realizaron en las habitaciones individuales de las pacientes ingresadas, donde solo estaban presentes en el momento de la entrevista la participante y la entrevistadora, evitando influencias de terceras personas.

Consideramos que al realizar la entrevista una mujer ha podido facilitar la realización de las entrevistas, como ha ocurrido en otros estudios de índole parecida, ya que el hablar entre mujeres permite exteriorizar pensamientos y opiniones que probablemente ante un entrevistador de sexo masculino hubiera estado condicionado, o como mínimo, limitado. Es importante a la hora de realizar una investigación ser conscientes de que el hecho de ser hombres o mujeres condiciona no sólo la manera de situarnos ante los temas, sino también la relación con la población de estudio y la accesibilidad a determinadas conversaciones y temas ${ }^{25}$.

\section{Limitaciones}

La limitación principal de este estudio ha sido el tiempo de las entrevistas. Estas no duraron más de 30 - 40 minutos. Resultó imposible volver a conseguir una segunda entrevista en el domicilio debido a las circunstancias personales de las mujeres. 


\section{Consideraciones éticas}

Este proyecto de investigación, tras ser valorado por el Comité de Ética de Investigación en Salud de la Provincia de Granada, ha recibido el visto bueno para su realización.

\section{RESULTADOS}

\section{Datos sociodemográficos (figura 2)}

\section{El deseo de la maternidad}

Ante la pregunta sobre el motivo que les impulsó a tener hijos/as, 2 mujeres dijeron que no fue buscado. Las otras 8 mujeres dijeron que había sido buscado con muchas ganas, que les apetecía y que siempre habían querido ser madres. Sin embargo, conforme avanzaban las entrevistas emergieron otras razones latentes que habían impulsado la maternidad en ese momento de sus vidas. Estos motivos que aparecieron fueron la edad, como reloj biológico que marca una fecha (véase tabla 2. Motivación por la edad), la integración en su medio social formados por amigos y hermanos con hijos, la idea de estar en una época de la vida donde toca tenerlos (basada en los primeros años de matrimonio para ellos y posteriormente, tener hijos/as es la continuación en su relación de pareja y trayectoria de vida). El segundo bebé, siempre fue buscado para dar un hermanito/a al primero y en todos los casos salvo 1, impulsado desde el deseo del hombre, no de la mujer. Las mujeres decían que con un hijo/a ya se habían sentido realizadas como madres.

\section{Mitos: instinto maternal, maternidad intensiva - ideal de buena madre y amor maternal.}

Solo una de las entrevistadas mencionó el concepto instinto materno viendo este como la responsabilidad que sentían ante su hijo. Sin embargo bajo la creencia inconsciente de instinto maternal se esconde los emociones de 
angustia, estrés y baja autoestima que manifestaban algunas mujeres. Estas sentían incertidumbre y confusión frente a los cuidados de su hijo/a, situación que se acentuaba con los múltiples y contradictorios consejos que suministraban familia y amigos. Esta situación las desbordaba, generando ansiedad y miedo ante la llegada al hogar sin el apoyo de los sanitarios (véase tabla 3. Miedo ante la crianza). También se observó, cómo las madres con experiencia previa (cuidado de sobrinos y/o hijos, $u$ experiencia profesional como maestras, enfermeras pediátricas,...) poseían unos conocimientos y habilidades previas que les proporcionaban mayor seguridad y confianza en sí mismas para afrontar la crianza.

Sobre la creencia de maternidad intensiva y la idea de buena madre observamos cómo las mujeres presentaron sentimientos de culpa y percepción de mala madre cuando tuvieron que incorporarse nuevamente al trabajo, sintiéndose menos culpables si pudieron dejar los hijos/as a cargo de un familiar. De las 3 mujeres que se quedaron sin trabajo durante el embarazo, una manifestó directamente como esta nueva maternidad le obligaba a renunciar a su vida profesional. Por este motivo, la mujer se pensó mucho si tener otro hijo/a, finalmente aceptó porque su marido deseaba mucho tener otro más. Tener que renunciar a su trabajo y su entorno laboral le generaba sentimientos de tristeza. En su entrevista manifestó la dificultad que tenía para conciliar trabajo y crianza: "yo estaba trabajando, y yo trabajaba en una panadería, y yo sé que con el otro niño, el horario, ya no me lo permite (...) y ya mira, ni trabajo ni na, a cuidarlos (...), con una (hija) lo llevaba mejor (el trabajo), pero ya con dos es que es imposible, no tenemos ninguna posibilidad" (Manuela, 35 años).

Todas las mujeres comentaron la dificultad y la falta de recursos para compatibilizar los hijos/as, la casa y el trabajo. Casi todas afirmaron la necesidad de la red de apoyo familiar para el cuidado de los hijos/as, sobre todo las que ya tenían algún hijo/a previo.

Por último, sobre la creencia del amor materno y "como una madre no hay nada", se observó como esta idea sustentaba el reparto desigual de los 
roles durante toda la crianza. Bajo esta creencia a los hombres se les valoró positivamente bajo el concepto de "hombre niñeros", siendo un valor positivo y atípico en los hombres. (María, 35 años: "mi mario me ayuda a to con el niño (...) él es que es muy niñero (...) tiene buena mano, le encanta, a él es que le gustan mucho los niños"). Sin embargo, esta cualidad fue vista como propia del sexo femenino. El no presentar este rasgo era crítica de mala madre o / y mujer poco "femenina". En las mujeres, no se consideró como un rasgo a valorar, porque se asumía que debía estar presente en todas.

En el estudio observamos como 2 mujeres definen a sus parejas como hombres niñeros por estar más involucrados con los pequeños. El resto de los 8 padres aludían al miedo, respaldados por sus mujeres (véase tabla 4 . Padres que aluden al miedo), para evitar los cuidados del bebé. Las 8 mujeres decían que sus parejas no estaban capacitados como ellas. Una de las mujeres afirmó que le gustaba esta situación: sentirme más capacitada que su marido, porque le daba mayor autoestima el hecho de "sentirse mejor" que los hombres en este campo.

\section{DISCUSIÓN}

El deseo de la maternidad, es un factor importante que puede condicionar la vivencia de la maternidad, dependiendo de cómo las mujeres prioricen la maternidad en sus vidas. Puede ser un factor protector, si proviene de un proceso de autodeterminación consciente, o un factor de riesgo si no existen motivos reales internos y propios de deseo. Ante la pregunta de por qué decidió tener un hijo/a, todas las mujeres (salvo 2 ) respondieron que siempre han tenido ganas y que siempre han querido tener niños/as sin saber qué más responder. Marjorie Devault ${ }^{25}$ observó que la aparición en las entrevistas de expresiones como el "ya sabes", los silencios, las dudas, los rodeos, ... muestran las dificultades para ofrecer discursos propios. En nuestro estudio las mujeres ante la pregunta qué les impulsó a tener hijos/as presentaron estas expresiones. Esto muestra la dificultad de las mujeres para explicar los motivos propios, más allá del discurso social habitual (edad, época 
de la vida e integración en el medio). Lo mismo se observó en otro estudio 20 donde se indica que las mujeres aprenden la maternidad "en base a una tipificación dentro del marco proporcionado por el contexto social y cultural de cada individuo, sin ser una condición interior de la conciencia individual". En nuestro estudio sólo dos mujeres expresaron un alto deseo de ser madres. Para ellas, la maternidad era una prioridad en sus vidas y pensaban acceder a ella ya fuera en pareja o como madres solteras.

En el estudio realizado por Cristina Palomar sobre "malas madres"1, se habla de un núcleo incognoscible en el deseo materno, relacionado con emociones, afectos y deseos poco explicitados y también por tradiciones, costumbres, normas y creencias que forman los discursos de género. Las mujeres, acceden a la maternidad de una manera mecánica, en base a unas tradiciones y llegadas a unos "patrones de normalidad". No son conscientes de la influencia que tienen los discursos sociales de género sobre ellas.

En el trabajo de Castilla ${ }^{20}$ podemos ver cómo desde la infancia las mujeres han incorporado un discurso sobre las prácticas, creencias y sentimientos que las mujeres deben poseer en su maternidad. Este esta impregnado fuertemente con componentes culpabilizadores y de sacrificio.

Tanto en nuestro estudio, donde algunas de las mujeres renunciaron a su vida laboral por su deber como madres, como en otros donde la mujer manifiesta que "de repente pasas a un segundo lugar, para todo. Has cogido el puesto de madre"26, donde el ideal de buena madre perpetúa a las mujeres a esa entrega total, en base a su esencia "natural", la cual no debe ser cuestionada $^{20}$. Esta visión y creencias hacen, como bien dice Molina ${ }^{23}$, un inicio a la maternidad como patología.

En la literatura científica nos encontramos estudios donde el matrimonio es entendido para traer descendencia27,28,29. Esto, también se refleja en nuestro estudio. Todas las mujeres, excepto dos, decidieron con sus parejas tener hijos e hijas. El motivo de tener descendencia fue argumentado porque pensaban que "les tocaba" ser madres por el momento que vivían y debido al 
tiempo que llevaban en pareja (asociando matrimonio con maternidad). Siempre previa a otras etapas de la vida y de la pareja. Ninguna fue consciente de las creencias que les impulsaban a ser madre, frente a un deseo propio de tener hijos/as. Sin embargo, todas excepto dos, afirmaron que no tendrían descendencia como madres solteras.

Si dejamos de lado los motivos que conducen a la maternidad y nos centramos en la edad, observamos como la edad de nuestras madres, al igual que la mayoría de las mujeres de la comarca rural que paren en el Hospital de Guadix, están dentro de la década de los treinta. En otro estudio ${ }^{3}$ se asocia la elección de esta década a cambios en factores sociales y culturales, como la emergencia de nuevos estilos de vida, la mayor reticencia de los jóvenes a comprometerse en la formación de una familia, la voluntad de las mujeres con estudios de plantearse la maternidad tras haber cumplido una serie de hitos en el terreno profesional o la exigencia de contar con una estabilidad económica.

También intervienen las dificultades de acceso a la vivienda, la precariedad de la ocupación, así como la carencia de servicios para las familias que faciliten la conciliación entre vida familiar y laboral $^{30}$. Estos factores coinciden con discursos de las mujeres entrevistadas. Una de las dos mujeres cuya prioridad era ser madre, dijo que debía haber logrado un desarrollo profesional y una estabilidad económica. Otras, comentaron la dificultad que suponía la maternidad con la conciliación de la vida familiar. Esta dificultad no se expresó como causa del retraso de la edad para tener hijos/as, sino como un factor que dificulta la vivencia de la maternidad.

Respecto a la maternidad intensiva y la vida laboral, existen estudios que visibilizan dicha dificultad $30,31,32$. Otros hablan de la sobrecarga que supone el trabajo remunerado y el no remunerado, en base a la "naturalización" de dichas labores como son la lactancia materna y los cuidados básicos del bebé ${ }^{20,23}$. Otros estudios muestran la dificultad para el éxito y la promoción laboral ${ }^{33,34}$. En nuestro estudio hubo varias mujeres que renunciaron a su trabajo para dedicarse a la maternidad y la crianza. La falta de recursos les impedía poder seguir con su trabajo. Renunciar a la vida laboral sólo se planteó 
en las madres, no en sus parejas. Esto concuerda con la creencia de que es la figura masculina el sustento económico principal de la familia ${ }^{22}$.

Otros mitos sociales en torno a la maternidad son, la asociación del conocimiento innato de las mujeres para cuidar a los hijos/as (instinto materno) y el amor materno instantáneo nada más nacer el bebé, frente a la realidad de la experiencia en la crianza y el establecimiento del vínculo materno progresivo.

La variable experiencia en la crianza muestra como las mujeres por ser mujeres no nacen sabiendo cómo se debe cuidar y criar un hijo/a, desmontando el mito del instinto maternal. Es la experiencia la que las capacita en estas tareas, a la vez que va forjando el vínculo entre ambos. Esto se ve en otro estudio ${ }^{26}$, donde la mujer manifiesta que "yo creo que pasa un tiempo hasta que vas conectando (...) tú sabes que es tu hija, que la quieres, pero hasta que los sentimientos esos se refuerzan, pasa un tiempo. Pasan meses". Las mujeres que fueron separadas de sus hijos/as al nacer manifestaron la dificultad de sentir estos como algo suyo, de ahí la importancia del contacto precoz, no solo por el bebé, sino por los progenitores y el establecimiento del vínculo familiar.

En relación a la creencia de que las mujeres tiene un conocimiento innato para cuidar a la descendencia, vemos como las madres con experiencias previas con niños presentan mayor seguridad y confianza en sí mismas y menor ansiedad ante los cuidados del bebé. Esto contrasta con las primerizas sin experiencia, que viven esos momentos con miedos. Datos que coinciden con otro estudio 35 .

Respecto a la imagen de "hombres niñeros", vemos como otras autoras como Mari Luz Esteban ${ }^{36}$ indican, que estas actitudes en ciertos hombres “no suponen cambios significativos en el reparto del trabajo reproductivo ni en las representaciones de la maternidad". Sólo se consigue limitar el proceso de maternidad y paternidad. La figura del varón es vital para un óptimo desarrollo infantil. Hay estudios que muestran como los hombres presentan alteraciones 
hormonales en su proceso de adopción al rol paterno ${ }^{37}$, sin embargo, la creencia del padre como papel secundario o como figura ausente sigue presente en la sociedad $22,38,39$.

Por último, la red de apoyo de nuestras madres la forman las figuras femeninas de sus familias, al igual que sucede en otros estudios $8,30,40,41$, sin embargo, estos estudios no reflejan el aspecto de que son sólo las mujeres, con la perpetuación de su rol femenino, las que suministran siempre los cuidados.

\section{CONCLUSIÓN}

El deseo de la maternidad esta influenciado por el discurso social y las condiciones socioculturales. Es importante visibilizar como hombres y mujeres están igualmente capacitados como padres y madres, siendo el asesoramiento de profesionales y el día a día el que van generando la experiencia necesaria para guiarles. En la actualidad estos mitos y creencias perpetúan un reparto desigual de roles de crianza, y una sobreexplotación o privación de los derechos y deberes reales de hombres y mujeres. El personal de enfermería que trabaja con futuras madres y padres debe ser consciente de las presiones y cargas que las creencias androcéntricas generan en las mujeres. Es importante ayudarlas a darse cuenta de las limitaciones y malestares que les generan esas creencias. El contacto cercano con las mujeres permite detectar en los estadios más tempranos los malestares que la maternidad androcéntrica genera en las nuevas madres. Es necesario generar nuevos roles que permitan una maternidad más saludable sin sobrecargas ni culpas, además de abrir la puerta a una paternidad digna para todos. Los hombres deben ejercer su papel, sin escudarse en miedos y sin ser privados por las creencias de su falta de capacitación. El personal de enfermería debe informar y formar de sus nuevas responsabilidades y capacidades a ambos progenitores. 


\section{BI BLI OGRAFí A}

1. Palomar Verea, Cristina. Malas madres: la construcción social de la maternidad. Debate feminista. 2004;30(15): 12-34.

2. Velasco Arias, Sara. Recomendaciones para la práctica del enfoque de género en programas de salud. [internet]. Madrid: Observatorio de la Salud de la Mujer. Dirección General de la Agencia de Calidad del Sistema Nacional de Salud, Ministerio de Sanidad y Política Social; 2009 [citado 1/10/2013]. Disponible

en: http://www.msssi.gob.es/organizacion/sns/planCalidadSNS/pdf/equidad/recomendVelasco2009.pdf

3. Saletti Cuesta, Lorena. Propuestas teóricas feministas en relación al concepto de maternidad. Clepsydra. 2008; 7: 169-83.

4. Alda, Carmen; Bayo-Borrás, Regina; Camps, Nuria; Cánovas i Sau, Gemma; Tubert, Silvia. et al. Figuras de la madre. Madrid: Ediciones Cátedra S.A.; 1996.

5. Badinter, Elizabeth. ¿Existe el amor maternal?: historia del amor materno: siglos XVII al XX. 2d ed. Barcelona: Ediciones Paidós Ibérica, S. A.; 1981.

6. Staneva, Aleksandra; Wittkowski, Anja. Exploring beliefs and expectations about motherhood in Bulgarian mothers: a qualitative study. Midwifery. 2013; 29:260-7.

7. Chávez-Courtois, Mayra; Hernández-Maldonado, Alejandra; Arce-Zacarías, Erika; Bolaños-Delfín, Ivett; González-Pacheco, Iztel; et al. Experiencia grupal de mujeres embarazadas y en etapa postparto, y su relación con la depresión y algunos factores sociales. Perinatol Reprod Hum. 2008;22(4):270-8.

8. Marín Morales, Dolores; Bullones Rodríguez, Mạ Ángeles; Carmona Monge, Francisco Javier; Carretero Abellán, Mạ Isabel; Moreno Moure, Mạ Amparo; et al. Influencia de los factores psicológicos en el embarazo, parto y puerperio. Un estudio longitudinal. Nure Investigación [internet]. 2008 [citado 20/11/2013]; Nov-dic: (37): 1-20.

Disponible

en: http://www.fuden.eu/FICHEROS_ADMINISTRADOR/INV_NURE/proyemb37210200893951.pdf

9. Mayberry, Linda J; Horowitz, June Andrews; Declercq, Eugene. Depression symptom prevalence and demographic risk factors among U.S. women during 
the first 2 years postpartum. JOGNN. 2007;36(6):542-9.

10. Póo, Ana Mạ ; Espejo, Claudio; Godoy, Claudia. Prevalencia y factores de riesgo asociados a la depresión postparto en puérperas de un centro de Atención Primaria en el sur de Chile. Rev Med Chile. 2008; 136:44-52.

11. Shamai, Michal; Kochal, Rinat-Billy. Motherhood starts in prison: the experience of motherhood among women in prison. Family Process. 2008; $47(3): 323-40$.

12. Finzi-Dottan, Ricky; Goldblatt, Hadass; Cohen-Masica, Orlee. The experience of motherhood for alienated mothers. Child and Family Social Work. 2012; 17: 316-25.

13. Ives, A; Musiello, T; Saunders, C. The experience of pregnancy and early motherhood in women diagnosed with gestational breast cancer. PsychoOncology. 2012;21:754-61.

14. Hiersteiner, Catherine. Narratives of low-income mothers in addiction recovery centers: motherhood and the treatment experience. Journal of social work practice in the addictions. 2004;4(2):51-63.

15. Muñoz, Luz Angélica; Sánchez, Ximena; Arcos, Estela; Vollrath, Antonia; Bonatti, Carla. Vivenciando la maternidad en contextos de vulnerabilidad social: un enfoque comprensivo de la fenomenología social. Rev. Latino-Am Enfermagem. 2013;21(4): [ 7 pantallas].

16. Sanders, Lorraine B. Women's voices: the lived experience of pregnancy and motherhood after diagnosis with $\mathrm{VIH}$. Journal of the association of nurses in aids care. $2008 ; 19(1): 47-57$.

17. Blegen, Nina Elisabeth; Hummelvoll, Jan Kare; Severinsson, Elisabeth. Experiences of motherhood when suffering from mental illness: a hermeneutic study. International J ournal of Mental Health Nursing. 2012;21:419-27.

18. Knowles, Mariska; Nieuwenhuis, Jan; Smit Brigitte. A narrative analysis of educators' lived experiences of motherhood and teaching. South African Journal of Education. 2009; 29:333-43.

19. Ferrell Fouquier, Katherine. The concept of motherhood among three 
generations of african american women. Journal of Nursing Scholarship. 2011;43(2): 145-53.

20. Castilla, Ma Victoria. La ausencia del amamantamiento en la construcción de la buena maternidad. Revista de estudios de género. La ventana. 2005; 22:189-218.

21. Palomar Verea, Cristina. Maternidad: historia y cultura. Revista de estudios de género. La ventana. 2005;22:36-67.

22. Rodríguez, María del Carmen; Peña, José-Vicente; Torío, Susana. La experiencia de la paternidad y la maternidad: análisis del discurso de las creencias sobre la crianza y el cuidado infantil. Infancia y Aprendizaje 2009; 32 (1): 81-95.

23. Molina, Ma Elisa. Transformaciones histórico culturales del concepto de maternidad y sus repercusiones en la identidad de la mujer. Psykhe. 2006; 15(2): 93-103.

24. Mercer T, Ramona. Nursing support of the process of becoming a mother. JOGNN 2006; 35:649-51.

25. Blázquez Rodríguez, Maria Isabel. Nosotras parimos ¿Nosotras decidimos en la atención sanitaria al embarazo, parto y puerperio?. la ed. Málaga: Servicio de publicaciones de la Universidad de Málaga; 2010.

26. De los Ríos Blázquez, Elena; Toribio Guijarro, Pilar. Relatando la maternidad. Lo tangible detrás de los estereotipos. Archivos de la memoria. 2012; 9(2).

27. Lara, Ma Asunción; Navarro, Claudia; Navarrete, Laura. Síntomas depresivos en el embarazo y factores asociados en pacientes de 3 instituciones de salud de la ciudad de México. Salud Mental. 2006;29(4).

28. Posso Quiceno, Jeanny Lucero. Las transformaciones del significado y la vivencia de la maternidad, en mujeres negras, indígenas y mestizas del suroccidente colombiano. Sociedad y economía. 2010(18):59-84.

29. Aramburú, Pilar; Arellano, Rosalyn; Jáuregui, Sandra; Pari, Lizbeth. Prevalencia y factores asociados a depresión posparto en mujeres atendidas en 
establecimientos de salud del primer nivel de atención en Lima Metropolitana, J unio 2004. Rev Peru epidemiol. 2008; 12(3): 1-5.

30. Maroto Navarro, Gracia; García Calvente, Mạ del Mar; Mateo Rodríguez, Inmaculada. El reto de la maternidad en España: dificultades sociales y sanitarias. Gaceta Sanitaria. 2004;18(2).

31. Goldbort, Joanne. Transcultural analysis of postpartum depression. MCN. 2006;31(2):121-26. González de Chávez, Asunción. Maternidad y paternidad: el ideal maternal. A tu salud . 2004;45(16):21.

32. Ruiz Cantero, Mạ Teresa; Vives Cases, Carmen; Papí Gálvez, Natalia; LaParra Casado, Daniel; Mateo Pérez, Miguel Ángel; et al. Indicadores para medir los determinantes de las desigualdades en salud desde la perspectiva del análisis de género. [internet]. Alicante: Departamento de Salud Pública, Área de Medicina Preventiva y Salud Pública, Universidad de Alicante; 2004. [citado 12/11/2013].

Disponible

en: http://rua.ua.es/dspace/bitstream/10045/22657/1/2004_Desigualdades_Salud.pdf

33. Solé, Carlota; Parella, Sonia. Nuevas expresiones de la maternidad. Las madres con carreras profesionales exitosas. RES. 2004;4:67-92.

34. Herman, Clem; Lewis, Suzan; Humbert, Anne Laure. Women scientists and engineers in european companies: putting motherhood under the microscope. Gender, work and Organization. 2013;20(5):467-78.

35. Lorén Guerrero, Laura; Millán Barreiro, Mạ Pilar. Vivencias y experiencias de la maternidad. Una revisión de relatos biográficos. Archivos de la Memoria. $2011 ; 8(3)$.

36. Esteban; Mari Luz. La maternidad como cultura. Algunas cuestiones sobre lactancia materna y cuidado infantil. En: Perdiguero, Enrique; Comelles, Josep M. Medicina y cultura. Estudios entre la antropología y la medicina. Barcelona: Ediciones Bellaterra; 2000. p. 207-226.

37. Brizendine, Louann. El cerebro masculino. $4 a$ ed. Barcelona: RBA divulgación; 2013.

38. Nieri, Liliana. Paternidad y maternidad: aproximaciones psicológicas y socioculturales. REV. POIESIS. 2012; 12(23): 1-12. 
39. Álvarez Nieto, Carmen; Linares Abad, Manuel; García Carriazo, Margarita. Actitudes de las parejas hacia la maternidad y la descendencia. SUMUNTÁN. 2007; 24:187-200.

40. Linares Abad, Manuel; Gálvez Toro, Alberto; Linares Abad, Miguel. La relación de ayuda a la mujer durante el embarazo, parto y puerperio del primer hijo. Index de Enfermería. 2002(38):9-14.

41. Kulig, Judith C; Wall, Margaret; Hill, Shirley; Babcok, Ruth. Creencias sobre la maternidad entre las mujeres menonitas que hablan bajo alemán. International Nursing Review. 2008; (55):420-26. 


\section{FIGURAS}

\begin{tabular}{|cl|}
\hline Figura 1. Guión entrevista. \\
\hline$\bullet$ & Deseo de la maternidad \\
\hline$\bullet$ & Vivencia del embarazo, parto y posparto \\
\hline$\bullet$ & Implicación de la figura paterna \\
\hline$\bullet$ & Red de apoyo social y familiar \\
\hline$\bullet$ & Vida laboral \\
\hline & Antecedentes personales y datos socio sanitarios \\
\hline
\end{tabular}

\begin{tabular}{|l|l|l|}
\hline $\begin{array}{l}\text { Figura 2. } \\
\text { Datos socio-demográficos de las 11 mujeres }\end{array}$ & \multicolumn{2}{|c|}{ Ninguna era cuidadora de discapacitados } \\
\hline Estado civil: todas casadas & $\begin{array}{l}\text { Edades: de 28 a 40 años. } \\
\text { (con 3 mujeres con 32 años y 3 con } \\
35 \text { años) }\end{array}$ & $\begin{array}{l}\text { Número de hijos/as: } \\
4 \text { mujeres primerizas, 6 mujeres con 2 } \\
\text { hijos/as y 1 con 3 hijas. }\end{array}$ \\
\hline $\begin{array}{l}\text { Nivel de estudios: } \\
2 \text { mujeres estudios primarios } \\
5 \text { mujeres estudios secundarios } \\
4 \text { mujeres estudios universitarios }\end{array}$ & $\begin{array}{l}\text { Situación laboral: } \\
5 \text { mujeres en paro (3 se quedaron } \\
\text { durante el embarazo) } \\
3 \text { mujeres no volverían a trabajar } \\
\text { hasta que los hijos/as fueran al } \\
\text { colegio. }\end{array}$ & $\begin{array}{l}\text { Hogar: } \\
9 \text { mujeres hacían solas las tareas del } \\
\text { hogar. } \\
\text { 2 compartían tareas del hogar con su } \\
\text { pareja, teniendo una de ellas } \\
\text { empleada del hogar. }\end{array}$ \\
\hline
\end{tabular}

\section{TABLAS}

Tabla 1. Figuras femeninas en el apoyo familiar

Manuela, 35 años: En el otro mi madre cuidaba a mi abuela que estaba en casa con ellos, y la tenía a cargo y vino al hospital, estuvo haciendo la comida, estuvo un día o dos y se fue, ahora se vino desde mis primeras correas y se queda ahora con nosotros unos días, porque murió mi abuela y mi padre ya no esta.

Cristina, 30 años: Mi madre es que se ha quedao hoy (...), nosotros vamos a comer al medio día a casa de mi madre siempre, y ya de las cenas me encargo yo.

Isabel, $\mathbf{3 3}$ años: Sí, siempre se viene una hermana unos días para ayudarme.

Martina, 32 años: Pues sí, todos echando una mano, mi madre, mi cuña,...

Gloria, 29 años: Sí, ahora tiene mi mario 15 días de permiso y luego se vendrá mi madre otros días.

\section{Tabla 2. Motivación por la edad.}

Carmen, 32 años: Es que llega un momento, la edad ¿no?. Lo requiere, es solamente eso.

María, 35 años: Más que na por la edad, porque cuando nació el otro yo tenía 30 años, y pues por no esperarnos más tiempo.

Susana, 35 años: No es que sea tarde ni nada, pero que a mi me hubiese gustado más joven, sí, porque yo pienso que ahora tengo 35 años, cuando tenga ella (su hija), por ejemplo, 35 y yo 70 y eso no me gusta.

Tabla 3. Miedos ante la crianza.

Manuela, 35 años: Estas tu tiempo sola con ella, y ya te vas adaptando, lo que vas haciendo un día mal, bueno mal, no tan bien, pues lo vas haciendo, y otro día mejor, y así, así vas cogiendo experiencia (...) las madres primerizas si es que estamos más perdías, ya no es lo mismo (con el segundo bebé), pero al principio sí que estas más perdía la verdad.

Cristina, 30 años: Yo le temía, porque me daba miedo coger un recién nacido (...), en el primero el miedo de si va a pasar algo, sino sabría cuidarla, no sabes, y bueno, ahora tampoco puedes saber, pero ya como tienes más experiencia.

Carmen, 32 años: Me ayudaban, es complicado, y cuando es el primero más todavía.

Esther, $\mathbf{4 0}$ años: El primero muy difícil, cuando yo en el hospital me la ponía no sabía cogerlo, y luego lo normal, yo que se, ya con el otro yo que se, y con este (la tercera hija), más chiquitina pero como has tenio los otros dos pues no es lo mismo que cuando tienes el primero. El primero siempre, como no tienes experiencia, te da más cosa.

Marta, 28 años: Un poco trastorna, porque mientras te habitúas a ellos, a comer, a bañarlo, a eso, pues un poco agobia.

Tabla 4. Padres que aluden al miedo.

Manuela, 35 años: Hasta el cordón se lo curé yo, que a mi mario le daba miedo.

Carmen, 32 años: Bueno, mejor, me ayuda más en la casa, y ya me ocupo yo de los niños (...), ya con ese si me maneja (hijo de 3 años), porque ya no tiene pañal y eso (...), lo ha cogiol(al bebé), porque no había otra pero que no, que no se hace, por lo menos me ayuda en mi casa, que hay hombres que ni eso y yo por lo menos no tengo queja. Esther, $\mathbf{4 0}$ años: Así, tan chiquititos no, cogerlo y eso sí, pero cambiarlos no. 